\title{
FLUTTER AND DIVERGENCE ANALYSIS USING THE GENERALIZED AEROELASTIC ANALYSIS METHOD
}

\author{
John W. Edwards ${ }^{1}$ and Carol D. Wieseman ${ }^{2}$ \\ NASA Langley Research Center, Hampton, Virginia 23681 USA \\ e-mail: j.w.edwards@larc.nasa.gov \\ ${ }^{2}$ e-mail: c.d.wieseman $(\tilde{a})$ larc.nasa.gov
}

Key Words: Aeroelasticity, Flutter, Divergence

\begin{abstract}
The Generalized Aeroelastic Analysis Method (GAAM) is applied to the analysis of three well-studied checkcases: restrained and unrestrained airfoil models, and a wing model. An eigenvalue iteration procedure is used for converging upon roots of the complex stability matrix. For the airfoil models, exact root loci are given which clearly illustrate the nature of the flutter and divergence instabilities. The singularities involved are enumerated, including an additional pole at the origin for the unrestrained airfoil case and the emergence of an additional pole on the positive real axis at the divergence speed for the restrained airfoil case. Inconsistencies and differences among published aeroelastic root loci and the new, exact results are discussed and resolved. The generalization of a Doublet Lattice Method computer code is described and the code is applied to the calculation of root loci for the wing model for incompressible and for subsonic flow conditions. The error introduced in the reduction of the singular integral equation underlying the unsteady lifting surface theory to a linear algebraic equation is discussed. Acknowledging this inherent error, the solutions of the algebraic equation by GAAM are termed 'exact.' The singularities of the problem are discussed and exponential series approximations used in the evaluation of the kernel function shown to introduce a dense collection of poles and zeroes on the negative real axis. Again, inconsistencies and differences among published aeroelastic root loci and the new, 'exact' results are discussed and resolved. In all cases, aeroelastic flutter and divergence speeds and frequencies are in good agreement with published results. The GAAM solution procedure allows complete control over Mach number, velocity, density, and complex frequency. Thus all points on the computed root loci can be matched-point, consistent solutions without recourse to complex mode tracking logic or dataset interpolation, as in the $\mathrm{k}$ and $\mathrm{p}-\mathrm{k}$ solution methods.
\end{abstract}

\section{INTRODUCTION}

Aeroelastic divergence of a restrained lifting surface or vehicle is a static stability problem that can be solved easily and accurately by eigenvalue methods for general, real matrices (e.g. pp. 431-440 of Ref. 1). The stability of an unrestrained vehicle, however, is a dynamic problem that cannot be solved accurately by static or quasi-steady methods. A number of recent publications have brought attention to differences and inconsistencies in calculations of the flutter and divergence behaviors of restrained airfoil ${ }^{2-4}$, unrestrained airfoil ${ }^{3,5,6}$, and cantilevered wing ${ }^{3,7,8}$ models when analyzed by dynamic stability methods. The use of a number of approximations to the unsteady airloads has led to this situation. Also, the various procedures of incorporating available, harmonic, unsteady airloads into the aeroelastic analyses, the methodologies of 'root-sorting' and 'mode-tracking' for reporting results, and the use of 'decay rates' as 'damping values' for nonoscillatory, real roots (with attendant 'bifurcations', 'jumps', and 'activation of lag roots') contribute to the differences. 
The unrestrained three Degree Of Freedom (DOH) airfoil model shown in Fig. 1 was studied by Rodden and Bellinger ${ }^{5}$ using the $\mathrm{p}$ and $\mathrm{p}-\mathrm{k}$ methods and is Example HA145A in Ref. 9. The case has also been studied by Chen ${ }^{3}$ using the $g$-method and by van Zyl ${ }^{6}$ using four forms of the p-k method. The restrained 2 DOF airfoil model (obtained by eliminating the 'fuselage' in Fig.1, or by letting the the fuselage mass approach infinity) was studied by Rodden and Bellinger ${ }^{2}$ using the $\mathrm{p}, \mathrm{p}-\mathrm{k}$, and $\mathrm{k}$ methods. The case has also been studied by $\mathrm{Chen}^{3}$ and by van $\mathrm{Zyl}^{4}$. Both cases have been studied for two locations of the center of gravity. For the forward location, the divergence speed, $U_{D}$, is lower than the flutter speed, $U_{f}$. Also, for the 3 DOF unrestrained case, a low frequency oscillatory mode instability appears instead of the well-known quasi-static divergence behavior. Since the mode "finds its origin in a tendency to static divergence," it was termed "dynamic divergence'. Small differences in the flutter and divergence speeds are reported for these cases. More significantly, differences are reported in the composition, origin, and continuity of the system modes for speeds well below and above $U_{D}$ and $U_{f}$, and for highly damped or undamped modes. Thus, there are discussions of speeds at which aerodynamic lag roots 'become active $^{3,10}$, speeds at which two damped, oscillatory modes merge and are no longer found for higher velocities ${ }^{4}$, and differences over the origin of the divergence $\operatorname{root}^{2,4-6}$ (i.e., whether it derives continuously from a structural mode or from an aerodynamic lag root).

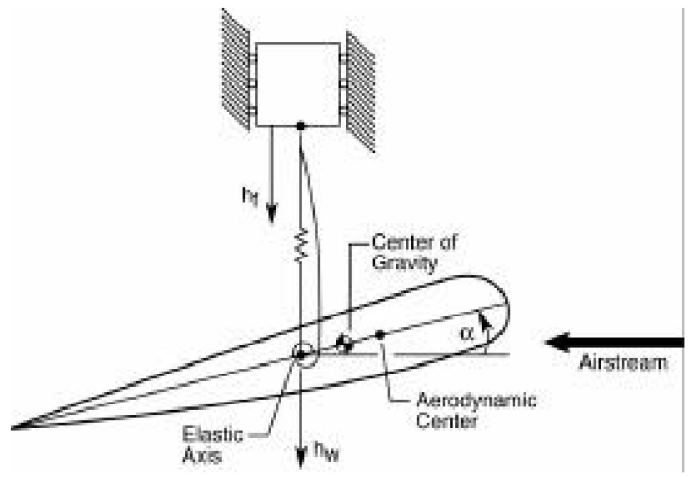

Figure 1. Three degree of freedom airfoil and 'fuselage.'

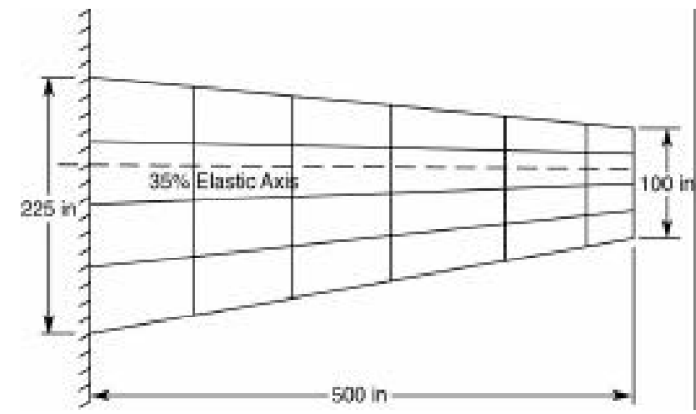

Figure 2. Transport (BAH) wing planform and aerodynamic strip modeling.

The jet transport wing model, the BAH wing, shown in Fig. 2 was introduced by Bisplinghoff, Ashley, and Halfman ${ }^{1}$ and has become a standard checkcase for flutter and divergence analysis of wings. It is Example HA145B in Ref. 9. Rodden and Stahl ${ }^{7}$ studied the BAH wing using the transient (p) method with airloads from strip theory and in Ref. 9 it is studied using the p-k method with the loads computed by the Doublet Lattice Method ${ }^{11}$ (DLM). The wing has also been studied by $\mathrm{Chen}^{3}$ using the g-method and by van $\mathrm{Zyl}^{8}$ using three forms of the $\mathrm{p}-\mathrm{k}$ method. There is agreement for the general structure of the instabilities: a bending-torsion flutter mode and a real divergence root. Again, small differences in $U_{D}$ and $U_{f}$ are noted, depending upon details of the calculations. Also, the discussions and differences noted above for the airfoil cases regarding the composition, origin, and continuity of the system modes are also present for the BAH wing case.

All of the above calculations have been based upon unsteady aerodynamic theories which assume purely harmonic structural oscillations. This leads directly to many of the issues underlying the results discussed above. A generalization of the harmonic oscillation 
assumption has been available and will be shown to resolve the issues and differences. Edwards $^{12-14}$ and Edwards, Ashley, and Breakwell ${ }^{15}$ proved the validity of the unsteady, linear, potential equation for arbitrary complex values of the Laplace transform variable, $s$, giving examples for Two-Dimensional (2-D) incompressible ${ }^{12,13,15}$, subsonic ${ }^{14}$, and supersonic $^{12,15}$ speeds and for Three-Dimensional (3-D) subsonic ${ }^{14}$ speeds. Examples of exact aeroelastic root loci for general complex values of $s$ illustrating flutter ${ }^{12,13,15}$ and divergence ${ }^{12,15}$ instabilities are given for 2-D incompressible and supersonic flow. For incompressible flow, it was shown that, above the divergenee specd, an additional pole (the divergence mode) is found on the positive real axis. The mode exists in addition to those deriving from the structural degrees of freedom. Edwards ${ }^{14}$ also showed that existing computer codes, developed for the calculation of harmonic oscillation airloads, could be generalized in a straightforward manner. A DLM computer code was generalized, and damped and undamped airloads were calculated for a swept and tapered wing. Cunningham and Desmarais ${ }^{16}$ and Cunningham ${ }^{17}$ performed similar generalizations of 2-D and 3-D unsteady potential theory Kernel Function Method codes for subsonic and supersonic flow, respectively. They present generalized airloads for an oscillating airfoil and generalized flutter analyses for several wing configurations.

The purpose of this paper is to present new analyses of the restrained and unrestrained airfoil models, and the BAH wing model using a Generalized Aeroelastic Analysis Method. Generalized unsteady airloads (incompressible and subsonic) are used to calculate the exact root loci of these models throughout the complex s-plane, including their flutter and divergence behaviors. Along with attention to the number and nature of the singularities involved, the new analysis gives a complete, intuitive explanation of the stability of the models and new insights into the capabilities and limitations of the computational methods in use.

\section{AEROELASTIC SOLUTION METHODS}

Aeroelastic stability analyses are based upon the Laplace transformed flutter equation

$$
\left[M_{s} s^{2}+B_{s} s+K_{s}-A(\bar{s}, M)\right]\{q(s)\}=0
$$

where $M_{s}, B_{s}$, and $K_{s}$ are the structural Mass, Damping, and Stiffness matrices and $A$ is the Generalized Aerodynamic Force (GAF) matrix which is a function of the generalized reduced frequency, $\bar{s}=s b / U$ and Mach number, $M$. Hassig ${ }^{18}$ summarizes aeroelastic solution methods which have become well known: the $\mathrm{p}$ method ('transient' method ${ }^{2,7}$ ), the k method ('American' method, 'U-g' method, ' $K$ ' and 'KE' methods in MSC/NASTRAN"), and the p-k method ('British' method, 'PK' method in MSC/NASTRAN'). All of these methods have been based upon the assumption that the required unsteady aerodynamic loads, $A$, are available, except in some special cases, only for purely harmonic oscillation of the structural degrees of freedom. References 12-15 show that this has been an unnecessary restriction and that airloads for arbitrary complex values of frequency, $\mathrm{p}$ (or $s=\sigma+i \omega$ herein), can be computed by suitably generalized computational methods and codes.

Returning to the development of solution methods, the $\mathrm{p}-\mathrm{k}$ method has been further differentiated by the treatment of aerodynamic damping. Rodden, Harder, and Bellinger ${ }^{19}$ introduced the $A$ matrix as 


$$
A=\left(\frac{1}{4} \rho \bar{c} U Q^{I} / k\right) p+\frac{1}{2} \rho U^{2} Q^{R}
$$

where $Q=Q^{R}+i Q^{I}$ is the matrix of GAFs for purely harmonic oscillations at a given reduced frequency $\bar{s}=i k=i \omega b / U$. In his ' $g$-method', Chen ${ }^{3}$ introduces the $A$ matrix as

$$
A=\frac{1}{2} p U^{2} Q^{\prime}(i k) g+\frac{1}{2} p \bar{c} U^{2} Q(i k)
$$

Finally, van $Z \mathrm{yl}^{8}$ uses a variation on the g-method,

$$
A=-\frac{1}{2} \rho U b\left(\frac{d(Q(i k))}{d k}\right) p+\frac{1}{2} \rho U^{2}\left(Q(i k)-k \frac{d(Q(i k))}{d k}\right)
$$

with special attention to numerical problems at $k=0$ by means of spline approximation of the elements of $Q$ and its derivative. The use of derivatives of the GAF matrix in Eqs. 3 and 4 makes these methods questionable for use in divergence analysis, with $k=0$. Subsonic airloads have a logarithmic branch point at the origin where their derivatives are undefined.

All of the checkcases to be considered are for incompressible flow, $M=0$. For the BAH wing model, the generalized DLM code described below is also directly applicable for subsonic, compressible flow and example calculations are given. For the 2-D airfoil cases the Q matrix loads are available in closed form (e.g., Eqs. 5-311,312 of Ref. 1). They involve the Theodorsen function, $C(i k)$, which can be utilized in tabulated form (for a series of reduced frequencies, $\mathrm{k}$, and with interpolation for intermediate values) or in approximate forms, such as that of W. P. Jones ${ }^{20}$ wherein the Wagner function is approximated by a two term exponential series curve fit

$$
\Phi(U t / b)=1-\sum_{n=1}^{2} \alpha_{n} \exp \left(-\beta_{n} U t / b\right)
$$

$C(i k)$ and $\Phi(U t / b)$ were known to be a Fourier transform pair, enabling the approximation of Eq. 5 to be incorporated into the flutter equation (1) as two additional linear, constant coefficient, 'lag' equations. These properties of the lag equations suggested that the approximation could be used for arbitrary complex values of reduced frequency, which is the essence of the $p$ method for flutter analysis.

As summarized by Rodden and Johnson ${ }^{9}$ these methods and approximations lead to forms of Eq. 1 which can be solved by linear matrix eigenvalue methods for general, real (Eq. 2) and complex (Eqs. 3 and 4) matrices. In the k-method, the introduction of structural damping, g, leads to an $\mathrm{NxN}$ matrix equation for the 'eigenvalue' variable $p^{2}=\left(-U^{2} /(1+i g)\right)=a_{n}+i b_{n}, n=1, \ldots, N$. Velocity and damping values are recovered as $U_{n}=\sqrt{-\left(a_{n}^{2}+b_{n}^{2}\right) / a_{n}}$ and $g_{n}=-b_{n} / a_{n}$. Interpretation of the results leads to complex mode tracking and root sorting logic and solution iterations are required in order to obtain consistent 'matched point' flutter solutions. The p-k method ( $\mathrm{p}$ method) leads to a $2 \mathrm{Nx} 2 \mathrm{~N}(2 \mathrm{Nx} 2 \mathrm{~N}+2)$ matrix equation for the variable $p=\omega(\zeta+i)$ where the viscous damping is $\varsigma=\frac{1}{2} g$. Real roots resulting from the lag equations in the p method, and the occurrence of real roots for the $\mathrm{p}-\mathrm{k}$ method, have led to the use ${ }^{9}$ of the decay rate of real, non-oscillatory roots as damping 
values in which $g$ is assigned the value $2 \operatorname{Re}(p) b / U(\ln 2)$. This facilitates some continuity in plots of the frequencies and dampings; however, many details of the resulting $U-g$ and $U-f$ plots are incorrect as will be shown below.

Ref. 21 discusses the problems of mode-tracking and mode-switching which surface in these aeroelastic solution methods and investigates two methods of improving solution performance by using information from the eigenvectors. Ref. 22 introduces a 'piecewise aerodynamic flutter method,' an alternative to the p-k method, in order to address problems with solution convergence and the difficult and time-consuming construction of state-space models.

\section{MODEL DESCRIPTIONS}

\subsection{Airfoil Models}

The transformed equations of motion for the 3 DOF airfoil of Fig. 1 are presented in Eq.6:

$$
\left\{s^{2}\left[\begin{array}{ccc}
1 & x_{\alpha} & 0 \\
x_{\alpha} & r_{\alpha}^{2} & 0 \\
0 & 0 & m_{f} / m_{w}
\end{array}\right]+s\left[\begin{array}{ccc}
-2 \zeta \omega_{h} & 0 & 0 \\
0 & -2 \zeta r_{\alpha}^{2} \omega_{\alpha} & 0 \\
0 & 0 & 0
\end{array}\right]+\left[\begin{array}{ccc}
\omega_{h}^{2} & 0 & -\omega_{h}^{2} \\
0 & r_{\alpha}^{2} \omega_{\alpha}^{2} & 0 \\
-\omega_{h}^{2} & 0 & \omega_{h}^{2}
\end{array}\right]\left(\left[\begin{array}{l}
h_{w}(s) \\
\alpha(s) \\
h_{f}(s)
\end{array}\right)=\frac{1}{\pi l}\left(\frac{U}{b}\right)^{2}\left(\begin{array}{c}
-c_{l}(s) b \\
c_{m}(s) \\
0
\end{array}\right)\right.\right.
$$

The bending and torsion springs are attached to a mass (fuselage), free to move vertically. Two examples are considered, differing only in the location of the center of gravity: in example 1 it is at $37 \%$ chord and in example 2 it is at $45 \%$ chord. Both examples assume incompressible flow. The aerodynamic center is at $25 \%$ chord and the elastic axis is at $40 \%$ chord. The chord is 6 feet, the nondimensional radius of gyration is $r_{\alpha}^{2}=0.25$, and the mass ratio $\mu=20$. The static un-balances are $x_{\alpha}=-0.06$ for example 1 and $x_{\alpha}=0.10$ for example 2. The uncoupled bending and torsion frequencies are $\omega_{h}=10 \mathrm{rad} / \mathrm{sec}$ and $\omega_{\alpha}=25 \mathrm{rad} / \mathrm{sec}$. Equal viscous damping of $\varsigma=\frac{1}{2} g=\frac{1}{2} 0.03$ is applied to the first two modes. The fuselage mass is assumed to be equal to the airfoil mass, $m_{f}=m_{w}$. The 2 DOF airfoil model is obtained from Eq. 6 by eliminating the row and column associated with the fuselage, $h_{f}$, keeping all the airfoil properties unchanged.

For incompressible flow, the airload coefficients in Eq. 6 are

$$
\left(\begin{array}{c}
-c_{l}(s) b \\
c_{m}(s)
\end{array}\right)=\left[M_{n c} \bar{s}^{2}+B_{n c} \bar{s}+K_{n c}+C(\bar{s}) R\left(S_{2} \bar{s}+S_{1}\right)\right]\left\{\begin{array}{l}
h_{w}(s) \\
\alpha(s)
\end{array}\right\}
$$

The matrices in Eq. 7 are defined in Ref. 13. The generalized Theodorsen function is

$$
C(\bar{s})=\frac{K_{1}(\bar{s})}{K_{0}(\bar{s})+K_{1}(\bar{s})} ; \quad \bar{s}=\frac{s b}{U}
$$

where $\mathrm{K}_{0}$ and $\mathrm{K}_{1}$ are modified Bessel functions which are defined and analytic throughout the $\bar{s}$-plane except for logarithmic branch points at the origin and at infinity. $\Lambda$ branch cut along 
the negative real axis makes them single-valued. Combining Eqs. 6 and 7 and defining the state vector $X^{T}=\left(h_{w}, \alpha, h_{f}, s h_{w}, s \alpha, s h_{f}\right)$, the equations of motion can be written as ${ }^{13}$

$$
\{s \mathbf{I}-A(s)\} X(s)=0
$$

where

$$
A(s)=\left[\begin{array}{cc}
0 & \mathbf{I} \\
M^{-1} K(s) & M^{-1} B(s)
\end{array}\right]
$$

Note that $K(s)$ and $B(s)$ are functions of $C(\bar{s})$. Thus the stability matrix, $A(s)$, has nonconstant, complex elements and attention must be paid to the logarithmic singularity at the origin. Thus, common eigenvalue methods of determining system stability using computer codes for real, gencral matrices cannot be applicd to Eq. 9. Instcad, a shareware family of routines for determining eigenvalues and eigenvectors of complex, general matrices was utilized, along with an eigenvalue iteration method to converge upon, and to track the singularities (e.g., poles) of Eq. 9. Thus, starting from an initial search location, $s_{n}$, the cigenvalues $\lambda^{T}=\left(s^{1}, \ldots, s^{6}\right)$ of $A\left(s_{n}\right)$ are determined and interrogated for the value of $s^{i}$ closest to $s_{n}$. An iterative relaxation method was used to converge upon a root of Eq. 9:

$$
s_{n+1}=s_{n}+r_{f a c}\left(s^{i}-s_{n}\right)
$$

In this manner, exact root loci of the modes of Eq. 9 are calculated by incrementing $U$ following the determination of a converged root. Typical starting locations are the coupled, wind-off structural frequencies. The method was very robust for the complex structural modes; relaxation factors of $r_{f a c}=0.8$ and velocity increments of $5 \mathrm{ft} / \mathrm{sec}$ were used. For studying behavior along the positive real axis, particularly near the origin, starting locations along the real axis were used with initial velocities selected to capture any potential real, divergence root. Smaller relaxation factors and velocity increments/decrements were also required there. For the airfoil examples, the convergence criterion was $\left|\left(s^{t}-s_{n}\right)\right|<0.001$ $\mathrm{rad} / \mathrm{sec}$. Since $A(s)$ is complex, the sets of eigenvalues, $\lambda\left(s^{i}\right)$, of $A\left(s_{n}\right)$ for any converged root, $s^{i}=\sigma^{i}+i \omega^{i}$, are not in general a collection of real roots and complex conjugate root pairs. Checks confirmed that, for complex valued roots, their complex conjugates, $\sigma^{i}-i \omega^{i}$, were also converged roots. Only the roots for the upper half $s$-plane are presented.

The use of another shareware computational routine for calculation of the determinant of a general, complex matrix was very useful in gaining understanding of the location and development of singularities. Thus, by evaluating $\operatorname{det}\{s \mathbf{I}-A(s)\}$ along a ray in the s-plane, the singularities (e.g., poles and zeroes) of the system characteristic equation can be studied. $\Lambda$ lso, by evaluating $\operatorname{det}\{s \mathbf{I}-A(s)\}$ along a circle contour about a test point, the number of singularities within the circle may be studied by noting the number of $2 \pi$ radian phase changes.

\subsection{Wing model}


The $\mathrm{B} \Lambda \mathrm{H}$ wing model shown in Fig. 2 consists of the truncated normal mode equations

$$
m_{i} \ddot{\xi}_{i}+2 m_{i} \zeta_{i} \omega_{i} \dot{\xi}_{i}+m_{i} \omega_{i}^{2} \xi_{i}=\frac{1}{2} \rho U^{2} \sum_{j=1}^{N} Q_{i, j} \xi_{j} ; i=1, N
$$

where $Q_{i, j}$ are elements of the $\mathrm{NxN}$ complex GAF matrix, $Q$. The mode shapes, masses, and frequencies of all ten modes, $N=10$, were extracted from the MSC/NASTRAN code. The viscous damping is zero for this case. Following transformation and identifying $X^{T}=\left(\xi_{1}, \ldots, \xi_{N}, \dot{\xi}_{1}, \ldots, \dot{\xi}_{N}\right)$, Eqs. 12 can be written in the same form as Eqs. 9 and 10 where $B(s)=0$ and $K(s)=K_{s}-\frac{1}{2} \rho U^{2} Q(\bar{s}, M)$. The GAF matrix, $Q$, was obtained from a modified version of the DLAT module of the ISAC program (Interaction of Structures, Aerodynamics, and Controls) described by Adams and Hoadley ${ }^{23}$. The original DLAT module implements the DLM code described in Ref. 24, calculating the GAF matrix, $Q\left(i k_{i n}, M\right)$, for purely harmonic reduced frequencies. The modified DLAT module calculates the GAF matrix for generalized reduced frequency, $\bar{s}$, in the manner described by Edwards ${ }^{14}$. Changes to the code are straightforward, with all occurrences of the real input reduced frequency variable, $k_{i n}$, replaced by the gencralized complex variable, $k=k_{i n}-i \sigma \frac{b}{U}$, signifying arbitrary $\bar{s}$-plane motion with complex reduced frequency $\bar{s}=\sigma \frac{b}{U}+i k_{i n}$. For example, in the DLM the kernel function of the integral equation to be solved involves functions such as Eq. A.26 in Ref. 24:

$$
I_{0}\left(u_{1}, k_{1}\right)=e^{i k_{1} u_{1}} \int_{u_{1}}^{\infty}\left[1-u\left(1+u^{2}\right)^{-1 / 2}\right] e^{-i k_{1} u} d u
$$

which is approximated by exponential series (Fq. A.30 in Ref. 24)

$$
I_{0}\left(u_{1}, k_{1}\right) \approx \sum_{n=1}^{11} \frac{a_{n} e^{-n c u_{1}}}{n^{2} c^{2}+k_{1}^{2}}\left(n c-i k_{1}\right)
$$

where the real constants $a_{n}$ and $c$ are given in Ref. $24, k_{1}=\omega r / U$, and the variables $u_{1}$ and $r$ are lengths related to the surface integration of the kernel. Generalization is accomplished by generalizing the real variable, $k_{1}$, to the complex variable, $k_{1}=(\omega-i \sigma) r / U=\left(k_{i n}-i \sigma \frac{b}{U}\right) r / b$. It is shown in Refs. 12, 13, and 15 that the stability of linear aeroelastic systems is determined by these generalized airloads. Portions of the system response which are not included (they are dependent upon initial conditions) do not affect system stability.

In the Appendix of Ref. 16, Cunningham and Desmarais discuss the singularities of the subsonic kernel function, showing that Eq. 13 has no singularities in the finite part of the $\bar{s}$ plane except for a logarithmic branch point at the origin. Similarly to the Theodorsen function, a branch cut along the negative real axis is required to make the function singlevalued. Now, the approximation of Eq. 14 has zeroes at $k_{1}=-i n c$ and poles at $k_{1}= \pm i n c$. Accounting for pole-zero cancellations, the approximation has poles located at $\bar{s}=-(b / r) n c, \quad n=1,11$ on the negative real axis in the $\bar{s}$-plane. Ref. 16 discusses the effect these poles on the performance of several approximations like that of Eq. 14. As $|\arg (s)-\pi / 2|$ increases towards $\pi / 2$, the approximations become unacceptable for determining accurate airloads. 


\section{RESULTS}

\subsection{Airfoil models}

The root loci for the 2 DOF restrained airfoil model are shown in Fig. 3 for the c. g. at 37\% chord and in Fig. 4 for the c. g. at $45 \%$ chord. The corresponding plots for the 3 DOF unrestrained airfoil model are shown in Figures 5 and 6 . Table I summarizes the flutter speeds, $U_{f}$, and frequencies, $\omega_{f}$, and the divergence speeds, $U_{D}$ (and frequencies, $\omega_{D}$, for the 3 DOF cases) for these cases. For the 2 DOF case, divergence is aperiodic with the divergence root emerging from the origin at $U_{D}$. For the 3 DOF case, the additional fusclage mass results in a low frequency oscillatory mode which becomes unstable at speeds near those of the restrained 2 DOF model divergence speeds. Rodden and Bellinger ${ }^{5}$ refer to this mode as a 'dynamic divergence' mode, which seems appropriate. All of these root loci were calculated using the eigenvalue iteration method of Eq. 11 and the exact Theodorsen airloads for arbitrary complex values of $\bar{s}$ and thus are the exact roots of the flutter equation (within the error band due to the convergence criterion). All of the published calculations of these characteristics (see Refs. 2-6, 8, and 9) are close to those given in the table and differences are due to the various approximations to the airloads (c.f. Eqs. 2-5) used by these authors. Of course this is as it should be, as these stability characteristics are defined by the airloads for purely harmonic oscillations which is the foundation of all of these approximate methods. It should be expected that deviations between the exact root loci of Figs. 3-6 and those calculated by the approximate methods would become progressively larger as root locations, $s$, become further removed from the vicinity of the $i \omega$ axis. The ' $\mathrm{x}$ ' symbols in Figs. 3-6 give the coupled, wind-off root locations. (The virtual mass airloads are included.) The root locus branch in Figs. 3-6 originating from the coupled torsion mode near $25 \mathrm{rad} / \mathrm{sec}$ behaves similarly in each case. The mode initially becomes damped for increasing speed along with decreasing frequency due to interaction with the lower frequency plunge mode. The mode eventually becomes the flutter mode and achieves a maximum level of negative damping before becoming asymptotic to a complex frequency value near the $i \omega$ axis. The root locus branch originating from the coupled plunge mode becomes monotonically more heavily damped in all cases. It never coalesces with its complex conjugate root to form real roots on the negative real axis. For both the $2 \mathrm{DOF}$ and $3 \mathrm{DOF}$ cases with $U=1000 \mathrm{ft} / \mathrm{sec}$, the root is located at $s=-100.87+i 30.89 \mathrm{rad} / \mathrm{sec}$ with the c.g. at $37 \%$ chord and at $s=-113.65+i 36.97 \mathrm{rad} / \mathrm{sec}$ with the c.g. at $45 \%$ chord.

The emergence of the divergence root as an additional, fifth root in the 2 DOF case was discovered by Edwards ${ }^{12}$. A simple explanation of this additional root is given by a low frequency model of a single degree of freedom pitching airfoil, which leads to the characteristic equation (c.f. Eq. 8-7 of Ref. 1)

$$
\left[1-C(\bar{s})\left(\frac{U}{U_{D}}\right)^{2}\right] \alpha(s)=0 \quad \text { wilh } \quad U_{D}^{2}=\frac{K_{\alpha}}{2 b^{2}\left(\frac{1}{2}+a\right) \frac{\partial c_{l}}{\partial \alpha}}
$$

"A pole of this aeroelastic system occurs at values of $s$ for which the coefficient in Fq. 16 is zero. Since $C(\bar{s})$ is purely real only on the positive real axis, poles can only occur there. Also, along the positive real axis, $C(\bar{s})$ decreases monotonically from a value of 1.0 at $|s|=0$. 


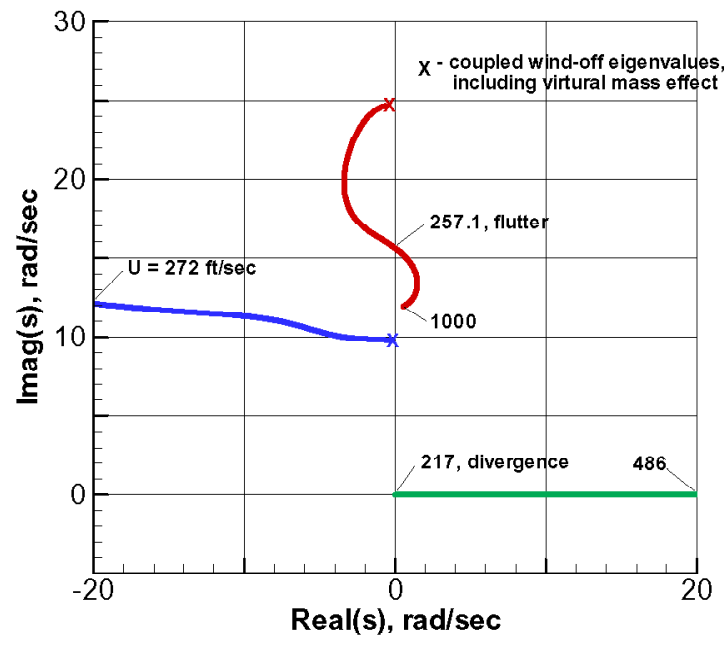

Figure 3. Exact root loci as a function of airspeed, showing flutter and divergence: two degrec of freedom airfoil, c. g. at $37 \%$ chord.

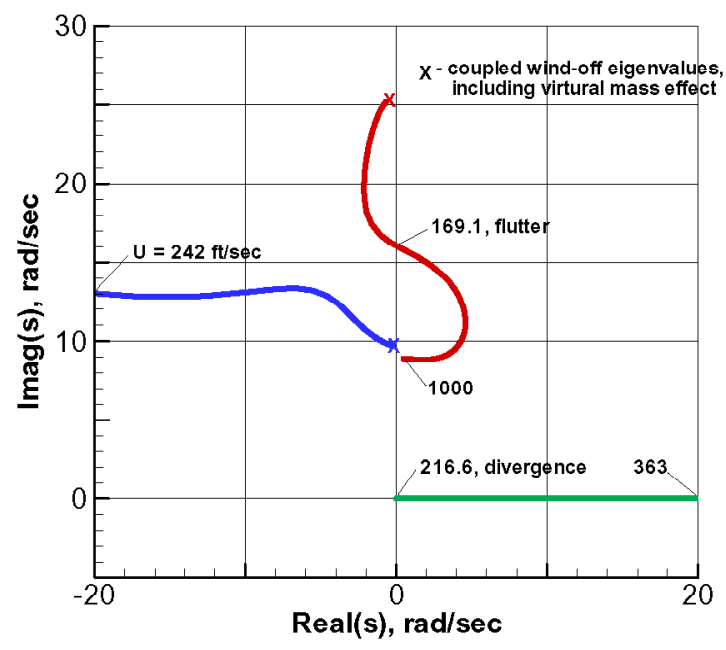

Figure 4. Exact root loci as a function of airspeed, showing flutter and divergence: two degree of freedom airfoil, c. g. at $45 \%$ chord.

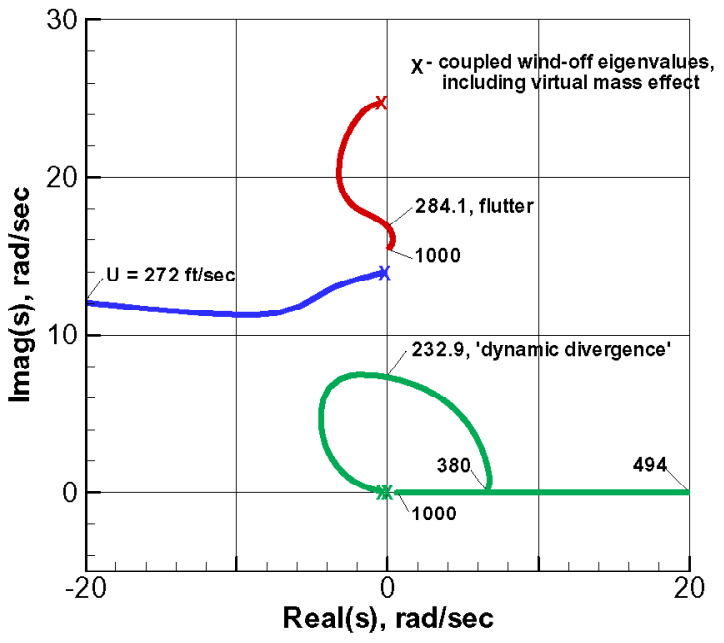

Figure 5. Exact root loci as a function of airspeed, showing flutter and divergence: three degree of freedom airfoil, c. g. at $37 \%$ chord.

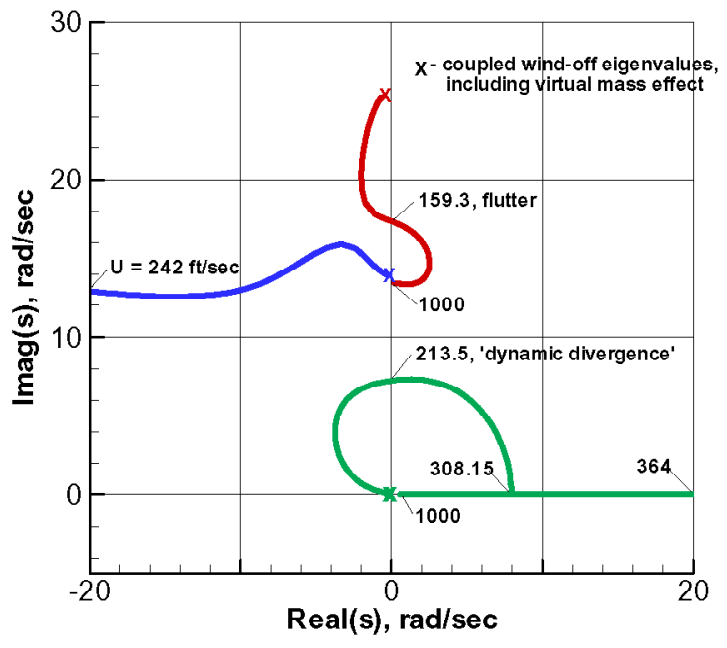

Figure 6. Exact root loci as a function of airspeed, showing flutter and divergence: three degree of freedom airfoil, c. g. at $45 \%$ chord.

to 0.5 at $|s|=\infty$. Hence, a pole cannot occur for $U<U_{D}$ and for $U>U_{D}$, only one real pole can occur. This mode produces the motion of the diverging airfoil and occurs in addition to the $2 \mathrm{n}$ structural poles." ${ }^{\prime 2}$ Another explanation of the possibility of additional roots is based on a fundamental theorem of algebra: an nth order constant coefficient polynomial equation (characteristic equation) has $\mathrm{n}$ and only $\mathrm{n}$ roots, but if the coefficients are not constant, as in Eqs. 1 and 9, no such guarantec exists.

The emergence of the divergence root at the origin for the 2 DOF case with the c.g. at $45 \%$ chord is illustrated in Fig. 7. The complex determinant of the stability matrix is evaluated along rays in the $s$-plane just above and just below the real axis at speeds bracketing the divergence speed, $U_{D}=216.6 \mathrm{ft} / \mathrm{sec}$. The development and emergence of the divergence pole 


\begin{tabular}{|l|l|l|l|l|}
\hline & \multicolumn{2}{|c|}{2 DOF } & \multicolumn{2}{c|}{3 DOF } \\
\hline c.g., $\% \mathrm{c}$ & 37 & 45 & 37 & 45 \\
\hline$U_{f}, \mathrm{ft} / \mathrm{sec}$ & 257.1 & 169.1 & 284.1 & 159.5 \\
\hline$\omega_{f}, \mathrm{rad} / \mathrm{sec}$ & 15.64 & 16.07 & 16.84 & 17.37 \\
\hline$U_{D}, \mathrm{ft} / \mathrm{sec}$ & 217.0 & 216.6 & 232.9 & 215.2 \\
\hline & & & 7.29 & 7.30 \\
$\omega_{D}, \mathrm{rad} / \mathrm{sec}$ & - & - & & \\
\hline
\end{tabular}

Table I. Flutter and divergence characteristics of restrained and unrestrained airfoil models. is clearly seen. The effect of the branch cut along the negative real axis is also evident in the jump in phase there. Figure 8 shows the complex stability determinant at a higher speed, $U=315 \mathrm{ft} / \mathrm{sec}$, with the divergence pole located at $s-+11 \mathrm{rad} / \mathrm{sec}$.

The ability of aeroelastic models utilizing state-augmentation to accurately predict static divergence speeds has become accepted. Models akin to that of Eq. 5, termed Rational Function Approximations $^{25}$ (RFA), generally employ a sequence of negative, real eigenvalues (roots) bracketing the frequency range of interest. The resulting additional roots have been termed 'aerodynamic lag roots" 7 . They tend to cluster

about the negative real axis and as speed increases towards $U_{D}$ the root closest to the origin migrates to the right, crossing onto the positive real axis at $U_{D}$. Edwards ${ }^{12}$ illustrates this for an airfoil model with stability behavior similar to that of the above 2 DOF model (with the c.g. at $37 \%$ chord).

The 3 DOF airfoil case of Figs. 5 and 6 presents a different situation regarding the number of singularities in the problem. Here the stability matrix (Eqs. 6 and 9) is $6 \times 6$ and at least six roots would be expected. However, the complex determinant shown in Fig. 9 indicates the presence of a seventh root at the low speed of $U=5 \mathrm{ft} / \mathrm{sec}$ for this case with the c.g. at $45 \%$ chord. An isolated singularity (pole) is evident at the origin, along with a pair of damped, complex conjugate roots which constitute the dynamic divergence mode at $U=232.9 \mathrm{ft} / \mathrm{sec}$ (see Fig. 6). Fig. 10 shows the presence of three real roots at the higher speed of $U=315 \mathrm{ft} / \mathrm{sec}$; the additional pole at the origin and the two poles resulting from the merger of the dynamic divergence mode poles on the positive real axis at $U=308.15 \mathrm{ft} / \mathrm{sec}$ (see Fig. 6 ). (The four other roots of the bending and torsion modes are not displayed in Figs. 9 and 10.) Comparison of Figs. 8 and 10, at the same speed, is instructive in illustrating the differences between the 2 DOF and 3 DOF airfoil cases. Physically, the seventh root at the origin accounts for a quasi-static airfoil motion with constant plunge velocity for this unrestrained airfoil case.

Further calculations, not shown, explored the transition from the 3 DOF model to the 2 DOF model by calculating root loci for increasing values of the fuselage mass, $m_{f}$, in Eq. 6 . As $m_{f}$ increases, the bending and torsion mode loci of the 3 DOF models of Figs. 5 and 6 approach those of the 2 DOF models of Fig. 3 and 4. Also, the 'oval' locus of the dynamic divergence mode near the origin becomes smaller, shrinking to the origin in the limit of infinite $m_{f}$, and the dynamic divergence speed approaches the static divergence speed of the 2 DOF model. Rodden ${ }^{26}$ also notes this behavior. Of course, in the limit of infinite $m_{f}$, the 3 DOF model with its 7 singularities becomes the 2 DOF model with its 5 singularities. 


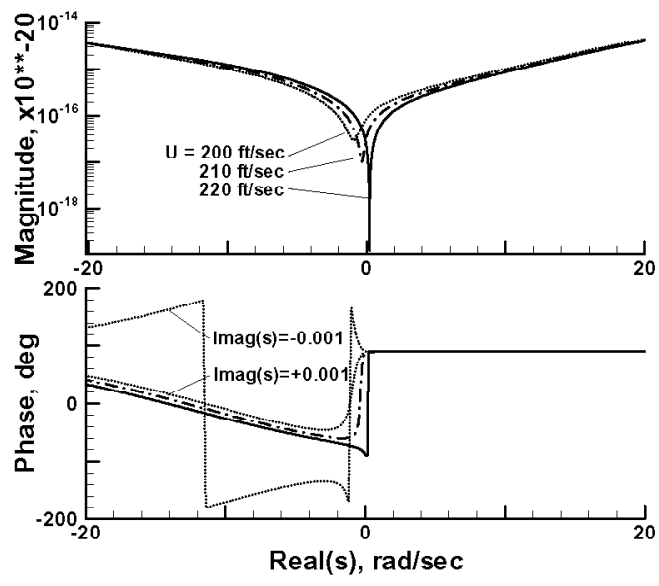

Figure 7. Determinant of stability matrix versus Real(s): two degree of freedom airfoil, c.g. at $45 \%$ chord.

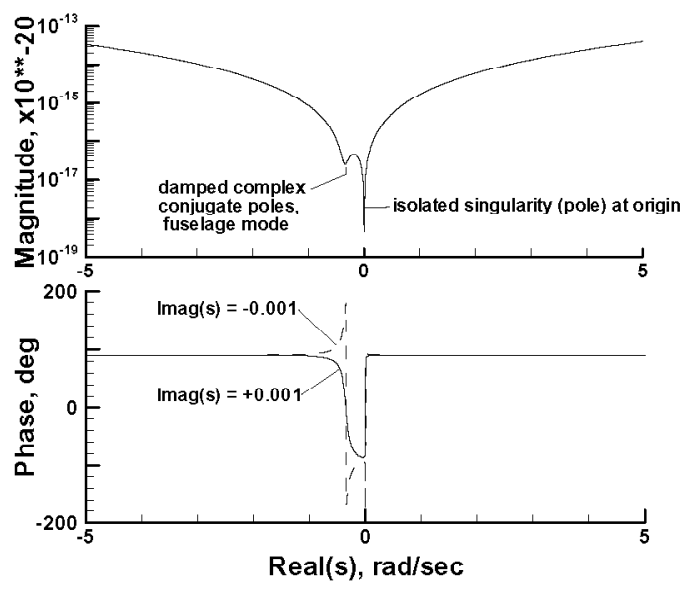

Figure 9. Determinant of stability matrix versus Real(s): three degree of freedom airfoil and fuselage, c.g. at $45 \%$ chord, $U=5 \mathrm{ft} / \mathrm{sec}$.

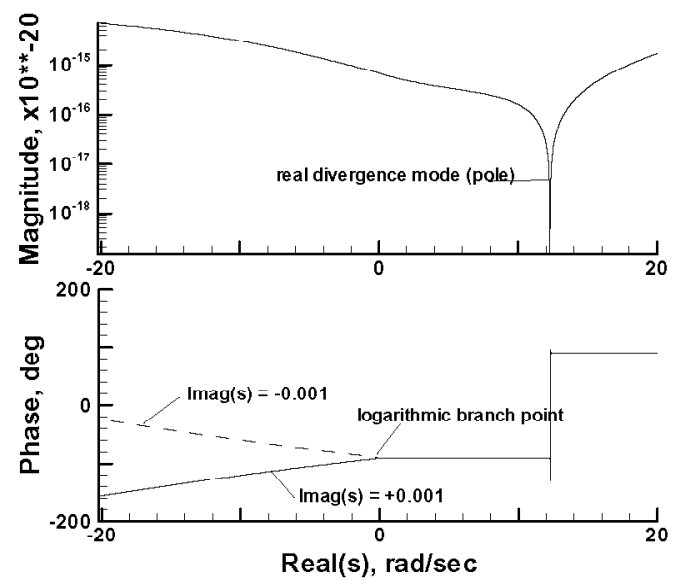

Figure 8. Determinant of stability matrix versus Real(s): two degree of freedom airfoil, c.g. at $45 \%$ chord, $U=315 \mathrm{ft} / \mathrm{sec}$.
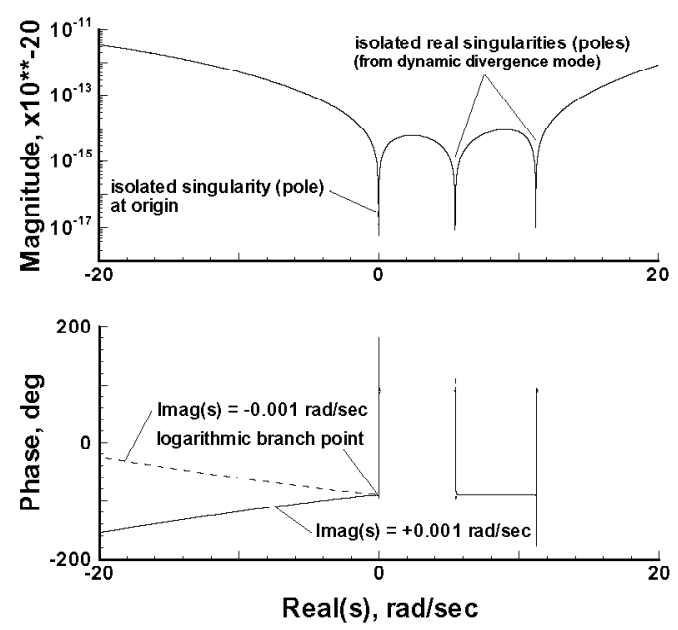

Figure 10. Determinant of stability matrix versus Real(s): three degree of freedom airfoil and fuselage, c.g. at $45 \%$ chord, $U=315 \mathrm{ft} / \mathrm{sec}$.

\subsection{BAH wing model}

For three-dimensional wing configurations, like the BAH wing, the stability matrix in Fq. 9 again contains non-constant elements, i. e. the airloads, $Q(\bar{s}, M)$. The same procedure described above for the airfoil cases was used to determine the root loci of the model. Ten modes were included resulting in a 20x20 general, complex stability matrix. The wind-off, coupled structural mode locations were used as starting values for the oscillatory roots. The velocity, for this $M=0$ case, was started at $100 \mathrm{ft} / \mathrm{sec}$ and incremented by 2 percent following convergence at each speed. The reference sea-level density is $\rho_{\text {ref }}=1.1468 \times 10^{-7} \mathrm{sl}(\mathrm{in}) / \mathrm{in}^{3}{ }^{3}$ In this case, the virtual mass in not included in the wind-off calculations, accounting for the small jump from the ' $x$ 's at the start of each locus. The iterative relaxation method of Eq. 11 was again very robust for the oscillatory modes and a relaxation factor of 0.80 was again 
used. For the real divergence mode root, speed increments or decrements of 0.5 percent and a relaxation factor of 0.02 were required. The convergence criterion was $\left|\left(s^{i}-s_{n}\right)\right|<0.03 \mathrm{rad} / \mathrm{sec}$.

The root loci for the first seven modes and the divergenee mode are shown in Fig. 11. The lowest flutter speed involves the second mode with $U_{f}=1059.7 \mathrm{ft} / \mathrm{sec}$ and $\omega_{f}=19.39$ $\mathrm{rad} / \mathrm{sec}$. A real, non-oscillatory root was found for $1450<U<2036 \mathrm{ft} / \mathrm{sec}$ with divergence occurring at $U_{D}=1647.25 \mathrm{ft} / \mathrm{sec}$. Flutter modes are also found at higher speeds for modes 3 , 5 , and 6 at $U=2449,2217(5280)$, and $6000 \mathrm{ft} / \mathrm{sec}$ respectively. For mode 5, looping of the locus produces two flutter speeds in the speed range covered. Such looping of the stability root loci is reminiscent of looping of airfoil load coefficients for lightly damped oscillations at high reduced frequencies shown by Cunningham and Desmarais ${ }^{16}$. Fig. 12 provides another viewpoint of the flutter instability from the determinant of the stability matrix versus $i \omega$ at $U=1056 \mathrm{ft} / \mathrm{sec}$, near the flutter speed. The presence of the flutter mode is clearly evident at $i \omega=i \omega_{f}$.

This example with its very coarse structural and aerodynamic paneling cannot be expected to yield high accuracy aeroelastic results, especially for the higher frequency modes and high values of reduced frequency. It is, however, a very good tutorial checkcase and has been well used to highlight differences obtained from alternative analyses. Fig. 11 illustrates that the 'exact' aeroelastic root loci for the oscillatory modes of this problem are well behaved, with no discontinuities, jumps, or bifurcations. (The present results are termed 'exact' in a sense that is discussed below.) Crossings of the loci do occur, but for very different speeds and lead to no ambiguity in interpreting the results, in contrast to the difficulties encountered in the $\mathrm{k}$ and p-k solution methods. ${ }^{21}$ It is the opinion of the authors that the $s$-plane presentation, with velocity as a parameter, gives a much clearer view of system stability than $U-g$ and $U-f$ diagrams. There is also no need to introduce decay rates as damping values for nonoscillatory real roots in order to interpret the plotted results.

An important result of this study is the insight gained of the behavior of the root loci along the real axis, particularly for the negative real axis. The loci of the first three oscillatory modes in Fig. 11 are shown approaching the negative real axis. Numerical convergence difficulties prevented further calculations for these loci and also for the locus of the real root for speeds $U<1450 \mathrm{ft} / \mathrm{sec}$. To understand the numerical issues involved, the determinant of the stability matrix versus $\operatorname{Re}(s)=\sigma$ was calculated for several velocities. Figs. 13 and 14 show the determinant for $U=1450$ and $U=1647.25\left(U_{D}\right) \mathrm{ft} / \mathrm{sec}$, respectively. The source of the numerical difficulty is immediately apparent in the numerous singularities (poles and zeroes) occurring along the negative real axis starting about $-7 \mathrm{rad} / \mathrm{sec}$. For $U=1450 \mathrm{ft} / \mathrm{sec}$, Fig. 13, an isolated pole has migrated out of the numerically difficult region, becoming the divergence mode, at $U_{D}=1647.25 \mathrm{ft} / \mathrm{sec}$, Fig. 14.

We return now to discuss the intent in referring to the current stability loci results as 'exact' results. The GAF matrix, $Q(\bar{s}, M)$, produced by the generalized DLAT module (the DLM code) contains the exact solutions for the linear matrix equation resulting from the several approximations utilized in the DLM formulation. The subsequent use of $Q(\bar{s}, M)$ in determining the root loci of the stability matrix of Eq. 9 throughout the $s$-plane is exact (a small error is due to the convergence criterion of the eigenvalue iteration method). Thus, any 


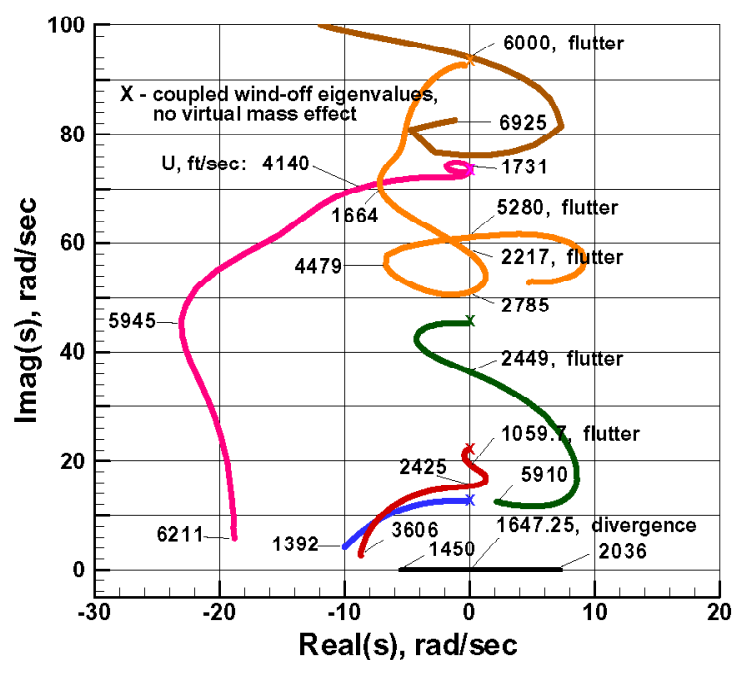

Figure 11. 'Exact' root loci as a function of airspeed showing flutter and divergence: transport (BAH) wing model, $M=0$.

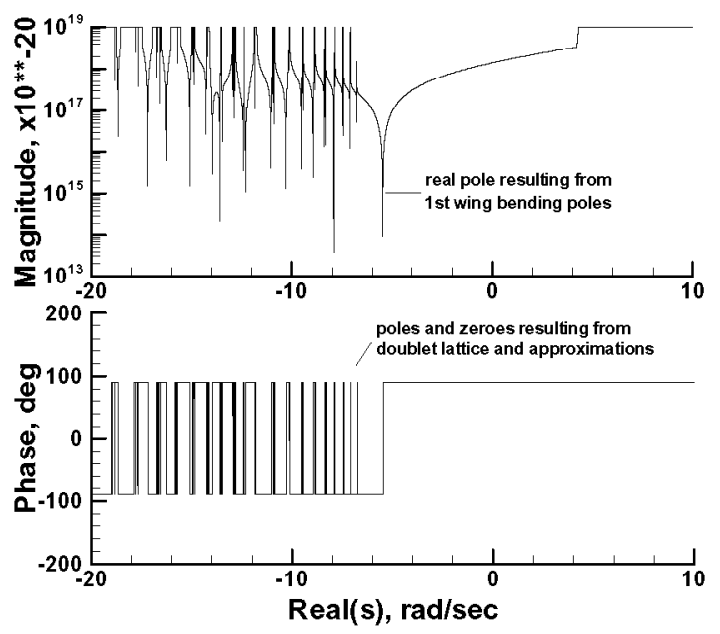

Figure 13. Determinant of stability matrix versus Real(s): transport (BAH) wing model, $U$ $=1450 \mathrm{ft} / \mathrm{sec}$.

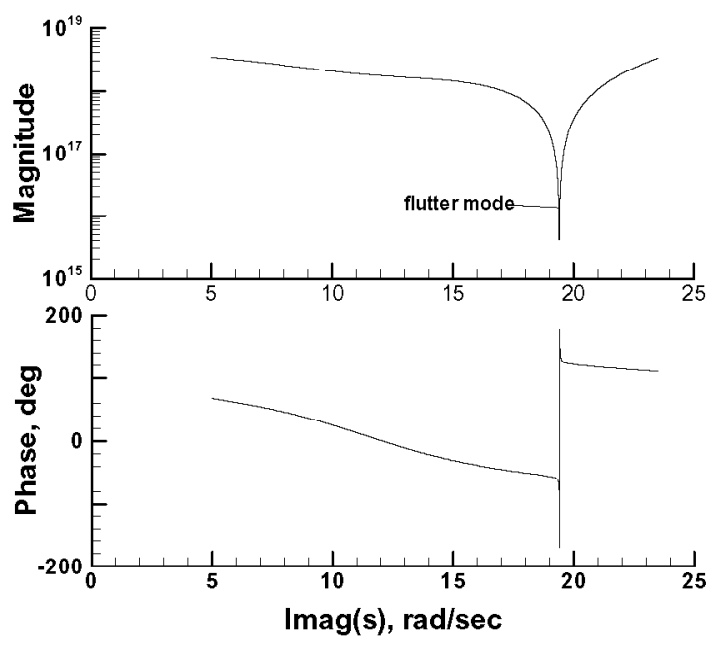

Figure 12. Determinant of stability matrix versus Imag(s): transport (BAH) wing model, $U$ $=1056 \mathrm{ft} / \mathrm{sec}$.

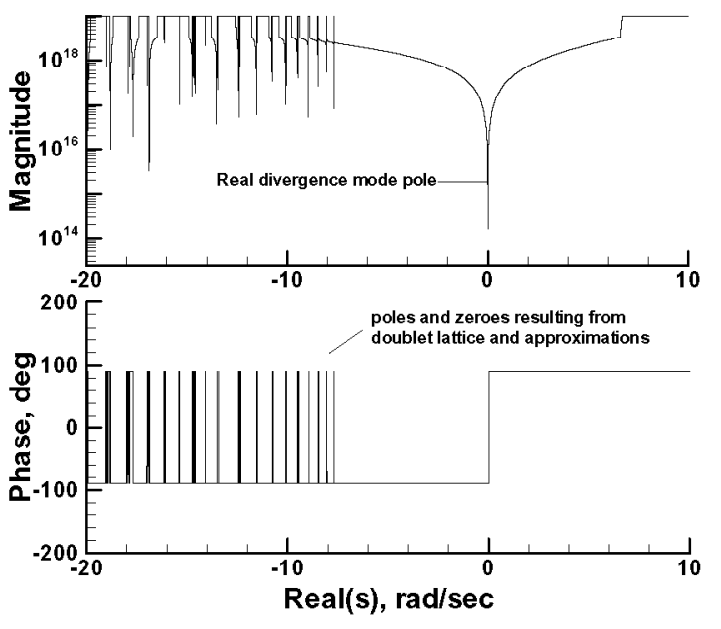

Figure 14. Determinant of stability matrix versus Real(s): transport (BAH) wing model, $\mathrm{U}$ $=1647.25 \mathrm{ft} / \mathrm{sec}$.

error involved in the current root loci calculations is that introduced by the reduction of the linear potential equation boundary value problem (BVP) to a linear matrix equation. The solution of the BVP is expressed as a singular integral equation ${ }^{11,16}$ involving a kernel function whose singular behavior has been discussed above ${ }^{16}$. Two main methods of solution of this integral equation are the Kernel Function Method (sec, c.g. Ref. 16) and the Doublet Lattice Method (Ref. 11). Both methods reduce the singular integral equation problem to a linear algebraic problem for the pressure loads on the surface and both methods use several approximations to accomplish this reduction. Both utilize exponential series similar to Eq. 14 
to approximate expressions like Eq. 13. The effect of this upon the singular behavior of the problem is to replace the logarithmic branch point singularity of the kernel function with isolated singularities (poles, or lag roots) typically located along the negative real axis as discussed above. Other approximations employed in the DLM are the discretization into the lattice of doublet lines and a parabolic approximation of the kernel along doublet lines. Approximations used in the Kernel Function Method are the truncation of the series approximation of the pressure distribution and the choice of the pressure loading functions to be used in the truncated series. It is with this understanding of the approximations introduced by the generalized DLM solution that the root loci computed by the current eigenvalue iteration method are termed 'exact'.

The similarity between RFA models and the exponential series approximation, Eq. 14, used in the DLM suggests that the singularities in Figs. 13 and 14 for $\operatorname{Re}(s)<-6 \mathrm{rad} / \mathrm{sec}$ derive from this series approximation. (The RFA given by the transform of Eq. 5 has negative, real poles as does Eq. 14.) Clusters of lag roots on and near the negative real axis similar to those seen in Figs. 13 and 14 are a well known feature in RFA flutter analyses.

These discussions of DLM solutions in the vicinity of the negative real axis lead naturally to the issue ${ }^{4}$ of the origin of the divergence mode in Fig. 11. Does the divergence mode derive from the bending mode or from a lag root? From Fig. 11 it is apparent that the bending mode and its complex conjugate root do merge onto the real axis at a speed slightly higher than $1392 \mathrm{ft} / \mathrm{sec}$ and that a real root does emerge from the cluster of roots at a speed slightly below $1450 \mathrm{ft} / \mathrm{sec}$. The change in the nature of the singular behavior of the kernel function resulting from the use of exponential approximations like Fq. 14 must also be acknowledged here. That is, the complication of the dense collection of poles and zeroes along the negative real axis is an artifact of the solution approximations, masking the behavior of the true exact solution in this region. Along with the above discussion of the inaccuracy of solutions for these highly damped values of complex frequency, this would appear to render the issue of the origin of the divergence root moot. In any case, the root has not been tracked for $1392<U<1450 \mathrm{ft} / \mathrm{sec}$.

The robustness of the GAAM eigenvalue itcration procedure in tracking the loci of the oscillatory structural modes of Fig. 11 was investigated. At issue is the maximum increment in velocity allowable in order to track the proper locus. For the two lowest frequency modes near 12 and $22 \mathrm{rad} / \mathrm{sec}$ all velocity increments up to $1000 \mathrm{ft} / \mathrm{sec}$ were able to track the loci over the range of velocity shown in the figure. For the third, fourth, and fifth modes near 46, 74 , and $94 \mathrm{rad} / \mathrm{sec}$ the maxima were 500,200 , and $100 \mathrm{ft} / \mathrm{sec}$ respectively. Of course these maximum increments are related to the closeness of the roots on the loci for corresponding velocities; c. f. the fourth and fifth mode loci near $s \approx-10+i 60 \mathrm{rad} / \mathrm{sec}$ for $U \approx 4500 \mathrm{ft} / \mathrm{sec}$.

Finally, the current Generalized $\Lambda$ eroelastic $\Lambda$ nalysis Method using the generalized DLM code is not restricted to the incompressible, $M=0$, condition of the BAH wing example. The main application of the DLM is in fact to lifting surface configurations in subsonic flow. To this end, root loci of the BAH wing model flutter mode versus Mach number were calculated. For this example, the sound speed is assumed to be $a_{\omega}=1500 \mathrm{ft} / \mathrm{sec}$ and $U=M a_{\omega}$. Fig. 15 shows the Flutter mode locus for two densities: the solid line is for the density of the above example and the dashed line is for a 50 percent higher density. The flutter Mach number (and velocity) decreases from 0.778 to 0.662 with increasing density. The symbols denote 0.10 Mach number increments. Note that each point on these loci are 'exact' matched point values of frequency and damping and are directly calculated by GAAM with no root- 
sorting, complex mode tracking logic, or dataset interpolation required.

\section{DISCUSSION}

All of these $(M=0)$ cases have been studied extensively by a number of researchers using various assumptions in the use of harmonic oscillation airloads for the several analysis methods described above. The 2 DOF airfoil cases have been analyzed in Refs. 2-4 and the 3 DOF cases have been analyzed in Refs. 3, 5, 6, and 9. The BAH wing model has been studied in Refs. 3, 7, and 8. There are many differences in solution details between the exact results given in Figs. 3-6, the 'exact' results given in Fig. 11, and those reported for these approximate solutions. Rather than focusing on each detail, the discussion to follow offers comments on the origin of these differences. All solutions produce good agreement for the flutter and divergence properties of the cases. This to be expected as all methods are based upon airloads which are accurate for purely harmonic oscillations (flutter and dynamic divergence) and for steady flow (static divergence). Differences in these properties are due solely to the differences utilized in these references for interpolating or approximating the oscillatory airloads. For speeds which are well removed from these flutter and divergence speeds and for highly damped or undamped modes, there are curious behaviors reported for the aeroelastic root loci (as shown in $U-g$ and $U-f$ plots). These include 'discontinuous roots', ${ }^{2,3}$, speeds for the 'activation of aerodynamic lag roots' 3,8 , 'bifurcations' of the roots ${ }^{3,4}$, inconsistency over the origin of certain of the roots ${ }^{4}$, and oscillatory roots ceasing to exist above critical speeds ${ }^{4}$.

These behaviors can be attributed to the use of harmonic oscillation airloads to infer loads for complex frequencies well removed from the $i \omega$ axis. Edwards ${ }^{12-15}$ proved that airloads derived for harmonic oscillation conditions can be used to derive airloads off the $i \omega$ axis by appealing to analytic continuation. There are, however, practical limits to this process. Regarding the airfoil cases, note that all of the exact loci shown in Figs. 3-6 are continuous and have no discontinuities or jumps. No lag roots become 'active' at any speed and the only type of root 'bifurcation' which occurs is for the dynamic divergence mode roots joining the real axis in Figs. 5 and 6. All of these types of features shown in Refs. 2-6, and 16 which differ from the corresponding root loci in Figs. 3-6 are spurious and are introduced by the airload modeling approximations used or by the aeroelastic solution methods.

A clarification of terminology is needed regarding use of the term 'bifurcation' to describe the merging of the dynamic divergence mode roots in Figs. 5 and 6 onto the positive real axis near $s \approx 6-8 \mathrm{rad} / \mathrm{sec}$. In nonlinear system theory, locations in parameter space at which a qualitative change in response occurs are termed bifurcation points. For the linear aeroelastic systems considered in this paper no such qualitative change in response occurs. These locations should be termed break-in (or breakaway) points. 
The issue of an additional real root emerging from the origin for restrained airfoil cases, Eq. 16, has also been addressed by $\mathrm{Heeg}^{27}$ who analyzed a $1 \mathrm{DOF}$ pitching airfoil model using an unsteady discrete vortex lattice method and eigenmode analysis. By varying the mass ratio, elastic axis, and radius of gyration, cases are shown in which the divergence mode is an additional eigenmode (as in Figs. 3 and 4) and in which the divergence mode results from the pitching mode root locus joining the real negative axis at a break-in point with subsequent divergent behavior. In the latter case, there would be no additional singularity. Ref. 27 also provides experimental verification of the former case from low speed wind tunnel model tests.

Regarding the BAH wing case (and by extension, three-dimensional lifting surface applications in general), the number of oscillatory frequencies for which the loads are available is limited in practice and can be insufficient to accurately define loads well removed from the $i \omega$ axis. Also, Ref. 16 shows that sin-gularities introduced by the exponential series, such as Eq. 14, in the kernel function solution lead to errors rendering the resulting 'exact' solutions unusable for significant regions of the $s$-plane. For a 12 -term series ${ }^{16}$ solutions for $|\arg (\bar{s})|<\pi / 4$ or $|\arg (\bar{s})|>3 \pi / 4$ become inaccurate or uneconomical, or both. An 11-term series $^{24}$ commonly used in DLM codes (and in the code used in the present study) was evaluated and found to be acceptable for $\arg (\bar{s})=5 \pi / 8$ but unacceptable for $\arg (\bar{s})=3 \pi / 4$. Thus, for the BAH wing all root loci in the vicinity of the negative real axis which are presented in Refs. 3, and 7-9 or shown in Fig. 11 have questionable accuracy.

Most of the curious behaviors of aeroelastic root loci mentioned above occur for heavily damped/undamped or real values of $s$ where this issue of accuracy of the airloads is most pronounced. On the other hand, the root loci of Fig. 11 obtained with the generalized DLM are the exact solutions to the lincarized algebraic problem resulting from the approximations to the kernel functions discussed above. The harmonic oscillation DLM airloads used in Refs. 3 , and 7-9 are all based upon this same linearized algebraic problem. Thus the 'exact' root loci of Fig. 11 can be used to evaluate corresponding solutions from these references. Fig. 11 shows that the 'exact' root loci are continuous and have no discontinuities or jumps. No bifurcations of roots occur and only one real root participates in the static divergence of the wing. Any differences among the 'exact' root loci of Fig. 11 and those from Refs. 3, and 7-9 are introduced by the airload modeling approximations or by the aeroelastic solution methods used in these references.

Desmarais and Rowe ${ }^{28}$ present alternative kernel approximation algorithms which are tailored to enable accurate approximations throughout the several regions of the complex plane. In place of exponential series like Eq. 14, the algorithms utilize Neuman series, continued fractions, asymptotic series, and Modified Functions. To the authors knowledge, they have not been implemented in an aeroelastic analysis program.

It is interesting to contrast this predicted behavior of the bending mode of the BAH wing model approaching divergence with that of the forward swept wing wind tunnel model described in Ref. 29. There, one of three cantilevered elastic models was lost due to static divergence while testing at $M=1.05$ and at 88 percent of the predicted divergence dynamic pressure. Significantly, the bending strain gage signal clearly indicated the presence of the 2 $\mathrm{Hz}$. bending mode throughout the event. It is possible that this event is similar to that shown in Fig. 3 where divergence occurs due to the emergence of an additional singularity at the origin while the bending mode remains distinct and oscillatory. 
Two main points resulting from the present investigation are: (i) the possibility of additional singularities occurring in aeroelastic analyses over and above those introduced by the structural dynamics and (ii) inherent limitations in computational codes commonly used for aeroelastic analyses for the study of behavior well removed from the $i \omega$ axis. It must be emphasized that only a small number of examples have been studied. No attempt has been made to delineate boundaries of parameters which exhibit the novel singular behavior shown in Figs. 3 and 4. The studies of Ref. 27 imply that sharp boundaries may not be found. Problems with the accuracy of the airloads for heavily damped wing motions notwithstanding, the BAH wing example appears to show that divergence for this case does occur subsequently to the bending mode frequency dropping to zero. It has been past practice to assume that this pattern must be involved in cantilevered wing divergence ${ }^{29}$. Results of this study (and the experimental results of Refs. 27 and 29) show that this assumption must be called into question. There is also the possibility of new insights which may be beneficial for subcritical divergence testing methods.

The following commentary provides further insight on issues dealt with herein: "An incorrect solution to an 'unrestrained divergence problem' was given in Ref. 10 using the static method and has been corrected in an errata ${ }^{30}$. In the errata, it was observed that in Ref. 10 the presumed instability occurred at a dynamic pressure at which the mean structural axis of the vehicle remained aligned with the freestream velocity vector rather than at the dynamic pressure of an actual physical structural instability. The incorrect quasi-static solution was applied to two example airfoils with an additional fuselage degree of freedom ${ }^{5}$ (c.f. the cases treated in Figures 5 and 6 herein) and compared with a dynamic solution. In one case the quasi-static divergence speed was lower by $1.7 \%$ than the dynamic solution; in the second case the difference was lower by $6.8 \%$. Since the errata had not yet been published, the explanation for the discrepancies offered ${ }^{5}$ was also in-correct. The conclusion of the errata was that 'divergence of an unrestrained vehicle should always be investigated by dynamic stability methods." (private communication, W. P. Rodden, July 2002) Dykman and Rodden $^{31}$ also treat the transient response of an unrestrained, flexible vehicle and compare the dynamic 'correct' solution with those obtained using modal residualization, modal truncation, and quasi-steady aerodynamics.

It must be emphasized that the use of the Generalized Aeroelastic Analysis Method for the calculation of aeroelastic root loci as illustrated in the above examples has not cast doubt on other methods of determining flutter and divergence characteristics (velocity and frequency). However, the ability of performing aero-elastic analysis without the necessity of root-sorting 9 , root-searching, lining-up", or reduced-frequency-sweep ${ }^{3}$ techniques is attractive. It is important to understand that each converged root locus value shown in Figs. 3-6, 11, and 15 is a 'matched-point' solution. By appropriate specification of density, Mach number, and speed direct calculations of such 'matched-point' aeroelastic root loci can be computed for constant altitude with varying Mach number and velocity, or for constant Mach number with varying altitude and velocity.

\section{CONCLUSIONS}

The Generalized Aeroelastic Analysis Method (GAAM) is applied to the analysis of three well-studied checkcases: restrained and unrestrained airfoil models, and a wing model. An eigenvalue iteration procedure is used for converging upon roots of the complex stability matrix. For the airfoil models, exact root loci are given which clearly illustrate the nature of the flutter and divergence instabilities. The singularities involved are enumerated, including an additional pole at the origin for the unrestrained airfoil case and the emergence of an 
additional pole on the positive real axis at the divergence speed for the restrained airfoil case. Inconsistencies and differences among published aeroelastic root loci and the new, exact results are discussed and resolved. The gencralization of a Doublet Lattice Method computer code is described and the code is applied to the calculation of root loci for the wing model for incompressible and for subsonic flow conditions. The error introduced in the reduction of the singular integral equation underlying the unsteady lifting surface theory to a linear algebraic equation is discussed. Acknowledging this inherent error, the solutions of the algebraic equation by GAAM are termed 'exact.' The singularities of the problem are discussed and exponential series approximations used in the evaluation of the kernel function shown to introduce a dense collection of poles and zeroes on the negative real axis. Again, inconsistencies and differences among published aeroelastic root loci and the new, 'exact' results are discussed and resolved. In all cases, aeroelastic flutter and divergence speeds and frequencies are in good agreement with published results. The GAAM solution procedure allows complete control over Mach number, velocity, density, and complex frequency, thus all points on the computed root loci can be matched-point, consistent solutions without recourse to complex mode tracking logic or dataset interpolation, as in the $\mathrm{k}$ and $\mathrm{p}-\mathrm{k}$ solution methods.

\section{ACKNOWLEDGEMENT}

The authors wish to acknowledge Dr. William P. Rodden of La Cañada Flintridge, California for his advice and discussions on the topics treated in the paper over the past quarter-century and for the commentary included above. The Aeroelasticity community has been well served in his development and/or advancement of these computational checkcases.

\section{REFERENCES}

[1] Bisplinghoff, R. L., Ashley, H., and Halfman, R. L., Aeroelasticity, Addison-Wesley, Reading, Mass., 1955.

[2] Rodden, W. P. and Bellinger, E. D., "Aerodynamic Lag Functions, Divergence, and the British Flutter Method," Journal of Aircraft, Vol. 19, No. 7, 1982, pp. 596-598.

[3] Chen, P. C., "A Damping Perturbation Method for Flutter Solution: The g-Method," Proceedings of the International Forum on Aeroelasticity and Structural Dynamics, Pt. 1, CP1999-209136/PT1, NASA, 1999, pp. 403-413.

[4] van Zyl, L. H., "Aeroelastic Divergence and Aerodynamic Lag Roots," Journal of Aircraft, Vol . 38, No. 3, 2001, pp. 586-588.

[5] Rodden, W. P. and Bellinger, E. D., "Unrestrained Aeroelastic Divergence in a Dynamic Stability Analysis," Journal of Aircraft, Vol. 19, No. 9, 1982, pp. 796-797.

[6] van Zyl, L. H., "Innrestrained Aeroelastic Divergence and the p-k Flutter Equation," Journal of Aircraft, Vol . 38, No. 3, 2001, pp. 588-590.

[7] Rodden , W. P., and Stahl, B. "A Strip Method for Prediction of Damping in Subsonic Wind Tunnel and Flight Flutter Tests," Journal of Aircraft, Vol. 6, Jan.-Feb. 1969, pp. 9-17.

[8] van Zyl, L. H., "Divergence and the Flutter Equation," Proceedings of the International Forum on Aeroelasticity and Structural Dynamics, Asociacion de Ingenieros Aeronauticos de Espana, Vol II, 2001, pp. 401-412.

[91 Rodden, W. P., and Johnson, E. H., 'MSC/NASTRAN Version 68 Aeroelastic Analysis User's Guide, MacNeal-Schwendler Corp., Los $\Lambda$ ngeles, C $\Lambda, 1994$.

[10] Rodden, W. P., "Aeroelastic Divergence of Unrestrained Vehicles," Journal of Aircraft, Vol. 18, No. 12, 1981, pp. 1072-1073. 
[11] Albano, E., and Rodden, W. P., " $\Lambda$ Doublet-Lattice Method for Calculating Lift Distributions on Oscillating Surfaces in Subsonic Flows," AIAA Journal, Vol. 7, No. 2, 1969 , pp. 279-285.

[12] Edwards, J. W., "Unsteady Aerodynamic Modeling and Active Aeroelastic Control," Stanford University SUDAAR 504, Feb. 1977.

[13] Fdwards, J. W., "Unsteady Aerodynamic Modeling for Arbitrary Motions," AIAA Journal, Vol. 15, Vo. 4, April 1977, pp. 593-595.

[14] Edwards, J. W., "Application of Laplace Transform Methods to Airfoil Motion and Stability Calculations," AIAA Paper No. 79-0772, St. Louis, MO, April 1979.

[15] Edwards, J. W., Ashley, H., and Breakwell, J. V., "Unsteady Aerodynamic Modeling for Arbitrary Motions," AIAA Journal, Vol. 17, No. 4, April 1979, pp. 365-374.

[16] Cunningham, H. J. and Desmarais, R. N., "Generalization of the Subsonic Kernel Function in the s-Plane, With Applications to Flutter Analysis," NASA TP-2292, March 1984.

[17] Cunningham, H. J., "Analysis of Preflutter and Postflutter Characteristics With MotionMatched Aerodynamic Forces," NASA TP 1232, July 1978.

[18] Hassig, H. J., "An Approximate True Damping Solution of the Flutter Equation by Determinant Iteration," Journal of Aircraft, Vol. 8, No. 11, 1971, pp. 885-889.

[19] Rodden, W. P., Harder, R. L., and Bellinger, E. D., "Aeroelastic Addition to NASTRAN," NASA CR 3094, 1979.

[20] Jones, W. P., "Aerodynamic Forces on Wings in Non-Uniform Motion," R and M 2117, 1945, Aeronautical Research Council.

[21] Eldred, M. S., Venkayya, V. B., and Anderson, W. J., "Mode Tracking Issues in Aeroelastic Analysis," AIAA Journal, Vol. 33, No. 7, 1995.

[22] Goodman, C., " $\Lambda$ ccurate Subcritical Damping Solution of Flutter Equation Using Piecewise Aerodynamic Function," Journal of Aircraft, Vol. 38, No. 4, July-August 2001, pp. 755-763.

[23] Adams, W. M. and Hoadley, S. T., "ISAC, A Tool For Aeroservoelastic Modeling and Analysis," NASA TM 109031, December 1993.

[24] Giesing, J. P., Kalman, T. P., and Rodden, W. P., "Subsonic Unsteady Aerodynamics for General Configurations. Part I, Volume I - Direct Application of the Nonplanar DoubletLattice Method," AFFDL-TR-71-5, Pt. I, Vol, I, U. S. Air Force, November 1971.

[25] Karpel, M. and Hoadley, S. T., "Physically Weighted Approximations of Unsteady Aerodynamic Forces Using the Minimum State Method," NASA TP 3025, March 1991.

[26] Rodden, W. P, Secondary Considerations of Static Aeroelastic Effects on HighPerformance Aircraft, Paper No.3 in Static Aeroelasticity in Combat Aircraft, AGARD Report No. 725, 1986.

[27] Heeg, J., "Dynamic Investigation of Static Divergence: Analysis and Testing," Ph. D. Dissertation, Duke University, Durham, North Carolina, May 2000.

[28] Desmarais, R. N. and Rowe, W. S, A Method for Computing the Kernel of the Downwash Integral Equation for Arbitrary Complex Frequencies, AIAA Paper No. 84-0983, May 1984.

[29] Ellis, J. W. Dobbs, S. K, and Miller, G. D., "Structural Design and Wind Tunnel Testing of a Forward Swept Fighter Wing," AFWAL-TR-80-3073, July 1980.

[30] Rodden, W. P., "Aeroelastic Divergence of Unrestrained Vehicles," Journal of Aircraft, Vol. 21, No. 1, January 1984, pp. 94-96.

[31] Dykman, J. R. and Rodden, W. P., Structural Dynamics and Quasistatic Aeroelastic

Equations of Motion, Journal of Aircraft, Vol 37, No. 3, May-June 2000, pp. 538-542. 